\title{
A Convex Approach to a Class of Non-convex Building HVAC Control Problems: Illustration by Two Case Studies
}

\author{
Ercan Atam*, Lieve Helsen \\ Dept. of Mechanical Engineering, KU Leuven, Celestijnenlaan 300 box 2421, Leuven 3001, \\ Belgium.
}

\begin{abstract}
In this paper, a convexification approach is presented for a class of non-convex optimal/model predictive control problems more specifically applied to building HVAC control problems. The original non-convex problems are convexified using a convex envelope approach. The approach is tested on two case studies: a benchmark building HVAC system control problem from the literature and control of a hybrid ground-coupled heat pump (HybGCHP) system. For the first application, convexified model predictive control was used and results were compared with fuzzy and adaptive control results. For the HybGCHP system, convexified optimal control was applied and the results were compared with dynamic programming based optimal control. In the first case superior performance was observed over the corresponding fuzzy and adaptive control results from the literature. For the HybGCHP system the associated convexified optimal control gave almost global optimal results in terms of responses and cost criteria. The suggested method is especially useful for optimal building HVAC control/design problems which include non-convex bilinear or fractional terms. Since a polynomial expression can be recursively expressed as a system of bilinear equations, the proposed method, in principle, can be applied to all systems where polynomial non-convexities exist.
\end{abstract}

Keywords: HVAC control, Hybrid ground-coupled heat pumps, Convex optimization, Convex envelope, Optimal control, Nonlinear model predictive control

\footnotetext{
*Corresponding author

Email address: Ercan. Atam@kuleuven. be (Ercan Atam)
} 


\section{Nomenclature}

Variables

$\mathrm{COP}$

$c_{e}$

$c_{g}$

$C_{a}$

$H_{t}$

$J$

$k$

$N_{c}$

$N_{p}$

$r_{f g}$

$r_{g s}$

$t$

$\dot{P}_{c h}$

$\dot{P}_{g b}$

$P_{h p}$

$\dot{P}_{p c}$

$\dot{Q}_{c}$

$\dot{Q}_{c h}$

$\dot{Q}_{\text {ext }}$

$\dot{Q}_{g b}$

$\dot{Q}_{\text {gain }}$

$\dot{Q}_{h}$

$\dot{Q}_{h p}$

$\dot{Q}_{i n j}$

$\dot{Q}_{n e t}$

$\dot{Q}_{p c}$

$T$

$\alpha_{g}$

$\eta_{g b}$
Description

coefficient of performance

electricity price

Unit

[-]

gas price

heat capacity

Euro/(kWh)

Euro/(kWh)

$\mathrm{kJ} / \mathrm{K}$

global heat transfer coefficient of the building enve- $\mathrm{W} / \mathrm{K}$ lope

total energy-use cost

Euro

conductivity

control horizon length

$\mathrm{W} /(\mathrm{mK})$

[-]

$[-]$

$\mathrm{cm}$

$\mathrm{cm}$

sec.

$\mathrm{W}$

W

W

electrical power used by heat pump

W

W

cooling load demand

$\mathrm{W}$

thermal power extracted from the building through active cooling

thermal power extracted from ground

W

thermal power supplied to the building by gas boiler W

internal gains

heating load demand

W

thermal power supplied to the building by the heat W

pump

thermal power injected to ground

W

net thermal power injected to ground

W

thermal power extracted from the building through W

passive cooling

temperature

diffusivity; exponent

gas boiler efficiency
${ }^{\circ} \mathrm{C}$

$\mathrm{m}^{2} / \mathrm{s} ;[-]$

[-] 


\section{Subscripts}

$\begin{array}{ll}\text { a } & \text { ambient air } \\ \text { aff } & \text { affine } \\ \mathrm{c} & \text { convex } \\ \mathrm{ch} & \text { chiller } \\ \mathrm{f} & \text { fluid } \\ \mathrm{fr} & \text { fractional } \\ \mathrm{g} & \text { grout } \\ \mathrm{gb} & \text { gas boiler } \\ \mathrm{hp} & \text { heat pump } \\ \mathrm{i} & \text { indoor air; inlet } \\ \mathrm{max} & \text { maximum } \\ \mathrm{min} & \text { minimum; minimize } \\ \mathrm{p} & \text { pipe } \\ \mathrm{pc} & \text { passive cooler } \\ \mathrm{s} & \text { soil }\end{array}$

\section{Abbreviations}

DP
GCHP
HVAC
HybGCHP
NMPC
OC
POD

\section{Introduction}

In the context of energy-efficient buildings HVAC control has gained increasing attention in recent years. Especially, future worries about the shortage of fuel sources and the requirement of reduction in greenhouse gas emission levels necessitate building HVAC control systems to be more efficient. HVAC devices and the building itself are often modeled using physical principles of heat transfer, thermodynamics and fluid mechanics. These models usually include nonlinearities and non-convexities which pose difficulties for controller design. Although it is not the aim to list all nonlinearities and non-convexities encountered in building HVAC control systems, among them the bilinear and fractional terms are the most ground-coupled heat pump

heating, ventilation and air conditioning hybrid ground-coupled heat pump nonlinear model predictive control optimal control proper orthogonal decomposition 
dominant ones. An example of a bilinear term in building HVAC applications is the mass flow rate times temperature. The coefficient of performance of a heat pump, which is the ratio of the thermal power delivered to the building over the electrical power used is an example of a fractional expression in building HVAC applications.

Once the building HVAC control system includes a bilinear or fractional term, the underlying system is a nonlinear system from the control point of view and it is a non-convex system from the optimization point of view. If the controller design is based on optimization (like optimal or model predictive control), then the controller design task basically involves solution of a non-convex optimization problem. It is very hard to solve non-convex control problems over longer control periods due to a large number of decision variables and the possibility of divergence. Even in case of a solution, a global minimum cannot be guaranteed. Existing solvers cannot handle non-convex optimization problems with a large number of decision variables. The simplest solution to such a non-convex control problem is to linearize the model around some operating point and using linear optimization. However, this leads to the risk of designing a non-working controller on the real system or a working controller with suboptimal results. As a result, linearization is not desirable and should be avoided whenever alternative controller design options are available.

A challenging HVAC control application where bilinear/fractional terms exist is the control of ground-coupled heat pumps (GCHP) and hybrid groundcoupled heat pump systems combined with low-exergy heat emission systems $[1,2,3,4,5,6,7,8,9,10]$. The attractivity of such systems comes from having the potential to reduce the primary energy use related to space heating and cooling by $70 \%$ compared to conventional heating and cooling systems [11]. For GCHP systems with vertical borehole heat exchangers (BHE), however, the large investment cost of the borefield represents a major bottleneck. This explains the trend towards compact, hybrid GCHP systems which combine smaller borefields with supplementary heating or cooling devices such as gas-fired boilers and chillers. Although the design of a compact HybGCHP system is often driven by cost considerations to limit the drilling cost without compromising thermal comfort in the building, sometimes other reasons may also lead to HybGCHP systems, such as limited drilling area for boreholes, the specific ground characteristics, regulation or too high imbalance of the thermal load.

De Ridder et al. and Verhelst $[12,5]$ used mathematical model-based control methods for HybGCHP systems, which allow global optimization. However, they are based on some simplifications and/or some unrealistic assumptions in- 
troduced during the controller design. For example, De Ridder et al. [12] used dynamic programming. Dynamic programming is a powerful method since it is a closed-loop, global optimal control algorithm. However, the model used by De Ridder et al. [12] for dynamic programming is a very simple first-order model for the ground mean temperature. The chosen control time step for the system is one week, which is very long since typical control actions for buildings may require control time steps in the order of minutes or hours. Moreover, the realization of the designed controller requires the measurement of the underground field temperature, for which measurement may be either difficult or non-accurate. As a result, the approach of De Ridder et al. [12] involves both some modeling simplifications and a hard-to-realize implementation. Verhelst [5] applied a linear optimal control method. The simplification made in this work is that the coefficients of performance (COP) for heat pump and chiller were taken to be constant, in contrast to being functions of source and sink temperatures. COP values were taken to be constant to avoid a non-convex optimization problem, which cannot be solved over an horizon of a couple years especially when short control time steps are considered. Although a mathematical model-based optimal control was considered, the simplifications of taking the mentioned COPs as constant values without a formal justification restricts the work of Verhelst [5]. Moreover, the model used for control and emulator were the same, which neglects the impact of model mismatch and therefore limits the generality of the approach followed.

The objective of this paper is to present and illustrate a convex relaxation method for a class of non-convex optimal control and non-convex model predictive control problems applied to two case studies, among which the control of a HybGCHP system to minimize the total energy cost is a special case. The convex relaxation method is based on the use of convex envelopes for bilinear and fractional terms. The convex envelope of a function is the largest convex function majorized by that function. Approximation of the non-convex terms by their convex envelopes will transform the optimization problem to an approximate problem which is convex and for which the global minimum can be found, if the problem is feasible. In convex optimization problems, a local minimum is a global minimum. Although the calculation of a convex envelope for a general multi-variable function is non-deterministic polynomial-time hard, there exist analytical formulas for a bilinear function or a rational function of two variables. Moreover, it is recursively possible to represent a polynomial non-convexity as a system of bilinear equations and hence the proposed convexification method, in principle, can be used for all systems where polynomial non-convexities exist.

Before testing the proposed convexification method on a HybGCHP system, 
the proposed convexified model predictive control approach was used on a benchmark building HVAC control system from the literature and the results were compared with the corresponding fuzzy and adaptive control results. Next, as the main application case study, HybGCHP system control was considered using the convexified optimal control approach and results were compared with dynamic programming control results (an approximate global optimal control algorithm) to assess the optimality of the convex envelope approach. The proposed approach gives promising results for both applications.

The rest of paper is structured as follows. Section 2 introduces the theoretical foundations and developments: introduction of a class of non-convex optimal and model predictive control problems, overview of convex optimization, introduction of convex envelopes for bilinear and fractional terms, convexification of the class of non-convex optimal and model predictive control problems through convex envelopes and finally a short summary of dynamic programming. Applications are considered in Section 3. As a first application, a benchmark building HVAC control system is considered from the literature. The second application, the main case study of this paper, is a HybGCHP system control. Finally, Section 4 , concludes with the main findings of this study.

\section{PART I: Theoretical Foundations}

In this part, first, we introduce a class of non-convex control problems which include the optimal/model predictive control of HybGCHP systems. Next, the proposed convex relaxation method for the given non-convex control problems is detailed. Since in Section 3.2 the proposed convex relaxation is applied to optimal control of HybGCHP systems and the results are compared with its dynamic programming-based control results, we also give a short overview of the dynamic programming at the end of this part.

\subsection{Non-convex Control Problem Classes}

In this section, we describe the general form of the two non-convex control problem classes that we convexify through the use of convex envelopes: nonconvex optimal control problems and non-convex model predictive control problems including bilinear/fractional terms. The optimal control and model predictive control of the HybGCHP system we consider in this paper are applications of these two classes of problems, as we mentioned before. 


\subsubsection{Non-convex Optimal Control Problem Class}

The general form of the non-convex optimal control problem class we consider is formulated as follows:

$$
\operatorname{minimize} \sum_{k=0}^{N} f_{c}(x(k), u(k), w(k))+f_{n c_{0}}\left(z_{1}(k), z_{2}(k)\right) g_{0}(w(k))
$$

subject to

$$
\begin{aligned}
& x(k+1)=A x(k)+B u(k)+g_{1}(w(k))+f_{n c_{1}}\left(z_{3}(k), z_{4}(k)\right) g_{2}(w(k)), \\
& f_{\text {eq-aff }}(x(k), u(k), w(k))+g_{3}(w(k))+f_{n c_{2}}\left(z_{5}(k), z_{6}(k)\right) g_{4}(w(k))=0, \\
& f_{\text {ieq-c }}(x(k), u(k), w(k))+g_{5}\left((w(k))+f_{n c_{3}}\left(z_{7}(k), z_{8}(k)\right) g_{6}(w(k)) \leq 0,\right.
\end{aligned}
$$

where $N$ is the length of the considered control period in terms of the number of control time steps, $x \in \mathbb{R}^{n_{x}}$ is the state vector, $u \in \mathbb{R}^{n_{u}}$ the control input vector, $w \in \mathbb{R}^{n_{w}}$ the measurable disturbance input vector, $z_{l} \in\left\{x_{i}, u_{j}: i=\right.$ $\left.1, \cdots, n_{x}, j=1, \cdots n_{u}\right\}$ for $l=1, \cdots, 8$. The functions $f_{c}, f_{\text {ieq-c }}$ are convex functions with respect to the variables $x, u, f_{\text {eq-aff }}$ is an affine function with respect to the variables $x, u, g_{l}, l=0, \cdots, 6$ are arbitrary continuous functions of $w$ and finally

$$
f_{n c_{l}}\left(z_{i}(k), z_{j}(k)\right)_{l=0, \cdots, 3}=z_{i}(k) z_{j}(k),\{i, j\} \in\{(1,2),(3,4),(5,6),(7,8)\}
$$

or

$$
f_{n c_{l}}\left(z_{i}(k), z_{j}(k)\right)_{l=0, \cdots, 3}=\frac{z_{i}(k)}{z_{j}(k)}, z_{i} \neq z_{j},\{i, j\} \in\{(1,2),(3,4),(5,6),(7,8)\} .
$$

As we see, the class of non-convex optimal control problems given by Eqs.(1)-(2) is a non-convex optimization problem class with bilinear or fractional non-convex expressions (of states/inputs) in the cost function and/or in the constraints.

\subsubsection{Non-convex Model Predictive Control Problem Class}

The non-convex model predictive control problem class we consider includes the same type of non-convexities as in Section 2.1.1 and can be regarded as a short optimal control problem solved at every control time step with the new data available at that time step:

$$
\operatorname{minimize} \sum_{k=i}^{i+N_{p}-1} f_{\mathrm{c}}(x(k), u(k), w(k))+f_{n c_{0}}\left(z_{1}(k), z_{2}(k)\right) g_{0}(w(k))
$$


subject to

$$
\begin{aligned}
& x(k+1)=A x(k)+B u(k)+g_{1}(w(k))+f_{n c_{1}}\left(z_{3}(k), z_{4}(k)\right) g_{2}(w(k)), \\
& f_{\text {eq-aff }}(x(k), u(k), w(k))+g_{3}(w(k))+f_{n c_{2}}\left(z_{5}(k), z_{6}(k)\right) g_{4}(w(k))=0, \\
& f_{\text {ieq-c }}(x(k), u(k), w(k))+g_{5}\left((w(k))+f_{n c_{3}}\left(z_{7}(k), z_{8}(k)\right) g_{6}(w(k)) \leq 0,\right.
\end{aligned}
$$

where $N_{p}$ is the prediction horizon and "i" is the current time step. The rest of descriptions of variables and functions are the same as in Section 2.1.1. In MPC control, the control input over the prediction horizon is calculated at every time step and the first element of the control input vector is applied at the current time step. At the next time step, the same calculation procedure is repeated. Due to this implementation scheme, MPC is sometimes called receding horizon control.

\subsection{Convex optimization and convex relaxation of bilinear/fractional terms}

In this section, before presenting a convex approximation of the non-convex control problems presented in Section 2.1, we start with the definitions of basic ingredients of convex optimization.

\subsubsection{Overview of convex optimization}

In words, a convex optimization problem is simply a convex function to be minimized over a convex set.

Definition 2.1 (Convex Set [13]). A set $S \subseteq \mathbb{R}^{n}$ is defined to be convex if for any $x, y \in S$ and $\theta \in \mathbb{R}$ with $0 \leq \theta \leq 1$, we have $\theta x+(1-\theta) y \in S$.

This means that if we take any two points in $S$ and draw a line segment between these two points, then every point on that line segment also belongs to $S$.

Definition 2.2 (Convex Function [13]). A function $f: \mathbb{R}^{n} \rightarrow \mathbb{R}$ is a convex function if

- its domain, $D(f)$, is a convex set.

- for any $x, y \in D(f)$ and $\theta \in \mathbb{R}$ with $0 \leq \theta \leq 1, f(\theta x+(1-\theta) y) \leq$ $\theta f(x)+(1-\theta) f(y)$.

This definition means that if we take any two points on the graph of a convex function $f$ and draw a straight line between them, then the portion of the function between these two points will lie below this straight line.

Armed with the above definitions of convex sets and functions, we are now ready to define a convex optimization problem. 
Definition 2.3 (Convex Optimization Problem: the most general form [13]). Given a convex function $f$, a convex set $S$ and the decision variable vector $x$, the associated convex optimization problem is defined as

$$
\begin{array}{r}
\text { minimize } f(x) \\
\text { subject to } x \in S .
\end{array}
$$

Definition 2.4 (Convex Optimization Problem: less general form [13]). Given a convex function $f$, convex functions $g_{i}$, affine functions $h_{i}$ and the decision variable vector $x$, the associated less general convex optimization problem is defined as

$$
\begin{aligned}
& \text { minimize } f(x) \\
& \text { subject to } \\
& g_{i}(x) \leq 0, \quad i=1, \cdots, p . \\
& h_{i}(x)=0, \quad i=1, \cdots, r \text {. }
\end{aligned}
$$

\subsubsection{Convex Envelope}

Next, the concept of convex envelope is defined.

Definition 2.5 (Convex envelope [14]). Given a continuous function $k(x)$, its convex envelope, denoted by conv $k(x)$, over a convex set $S$ is defined as the pointwise supremum of all convex functions which are majorized by $k(x)$ :

$$
\text { conv } k(x)=\sup \{r(x) \mid r \text { convex and } r(y)<k(y) \quad \forall y \in S\} .
$$

In other words, conv $k$ is the largest convex function such that conv $k(x) \leq k(x)$ for $x \in S$.

A positive bilinear function $f\left(x_{1}, x_{2}\right)=x_{1} x_{2}$ has the convex envelope

$$
\operatorname{conv} f\left(x_{1}, x_{2}\right)=\max \left\{a_{1} x_{2}+b_{1} x_{1}-a_{1} b_{1}, a_{2} x_{2}+b_{2} x_{1}-a_{2} b_{2}\right\}
$$

over the rectangular region $S=\left[a_{1}, a_{2}\right] \times\left[b_{1}, b_{2}\right]$, which is called McCormick envelope [14]. Similarly, the negative bilinear function $f\left(x_{1}, x_{2}\right)=-x_{1} x_{2}$ has the convex envelope [14]

$$
\operatorname{conv} f\left(x_{1}, x_{2}\right)=\max \left\{-a_{2} x_{2}-b_{1} x_{1}+a_{2} b_{1},-a_{1} x_{2}-b_{2} x_{1}+a_{1} b_{2}\right\}
$$

over the rectangular region $S=\left[a_{1}, a_{2}\right] \times\left[b_{1}, b_{2}\right]$. 
Using Eqs.(8) and (9), the equality constraint $w=f\left(x_{1}, x_{2}\right)=x_{1} x_{2}$ is convexified by

$$
\begin{aligned}
& w \geq a_{1} x_{2}+b_{1} x_{1}-a_{1} b_{1}, \\
& w \geq a_{2} x_{2}+b_{2} x_{1}-a_{2} b_{2}, \\
& w \leq a_{2} x_{2}+b_{1} x_{1}-a_{2} b_{1}, \\
& w \leq a_{1} x_{2}+b_{2} x_{1}-a_{1} b_{2} .
\end{aligned}
$$

Similarly, there exists an analytical convex envelope for the function $f\left(x_{1}, x_{2}\right)=$ $\frac{x_{1}}{x_{2}}$ over a positive rectangle in the first quadrant $S=\left[a_{1}, a_{2}\right] \times\left[b_{1}, b_{2}\right]$. In this case, the analytical formula for the convex envelope is given as [15]

$$
\begin{gathered}
\operatorname{conv} f\left(x_{1}, x_{2}\right)=\frac{b_{1}-x_{1}}{b_{1}-a_{1}} \frac{a_{1}}{\max \left\{a_{2}, \frac{b_{2}-x_{2}}{b_{1}-x_{1}}\left(a_{1}-x_{1}\right)+x_{2}, \frac{x_{2} \sqrt{a_{1}}\left(b_{1}-a_{1}\right)}{\left(b_{1}-x_{1}\right) \sqrt{a_{1}}+\left(x_{1}-a_{1}\right) \sqrt{b_{1}}}\right\}} \\
+\frac{x_{1}-a_{1}}{b_{1}-a_{1}} \frac{b_{1}}{\min \left\{\frac{x_{2}-a_{2}}{x_{1}-a_{1}}\left(b_{1}-x_{1}\right)+x_{2}, b_{2}, \frac{x_{2} \sqrt{b_{1}}\left(b_{1}-a_{1}\right)}{\left(b_{1}-x_{1}\right) \sqrt{a_{1}}+\left(x_{1}-a_{1}\right) \sqrt{b_{1}}}\right\}} .
\end{gathered}
$$

To obtain the convex relaxation of the constraint $w=\frac{x_{1}}{x_{2}}$, first, a similar convex envelope expression for the negative function $w=-\frac{x_{1}}{x_{2}}$ is obtained over $S$. Then, equivalent relations of $w \leq \frac{x_{1}}{x_{2}}$ and $w \geq \frac{x_{1}}{x_{2}} \Leftrightarrow-w \leq-\frac{x_{1}}{x_{2}}$ are utilized for $w=\frac{x_{1}}{x_{2}}$ and then $\frac{x_{1}}{x_{2}},-\frac{x_{1}}{x_{2}}$ are replaced by their convex envelopes. The overall system of relaxed constraints consists of 18 scalar convex constraints, which can be compactly represented as

$$
E_{\mathrm{fr}} w \geq g_{\mathrm{fr}-\mathrm{c}}\left(x_{1}, x_{2}\right)
$$

where $E_{\mathrm{fr}}$ is a constant vector and $g_{\mathrm{fr}-\mathrm{c}}\left(x_{1}, x_{2}\right)$ is a convex function with respect to the variables $x_{1}, x_{2}$. The derivation, although simple, is lengthy and hence was skipped.

\subsection{Convexified Optimal/Model Predictive Control Problems}

The convex relations of both non-convex optimal control and non-convex model predictive control problems given in Section 2.1 are obtained by defining the functions

$$
w_{l} \triangleq f_{n c_{l}}\left(z_{i}(k), z_{j}(k)\right), l=0, \cdots, 3
$$


and then replacing $f_{n c_{l}}\left(z_{i}(k), z_{j}(k)\right)$ with $w_{l}$ in the related equations and finally replacing the constraints $w_{l}=f_{n c_{l}}\left(z_{i}(k), z_{j}(k)\right)$ with the corresponding constraints in Eq.(10) or in Eq.(12), depending on whether $f_{n c_{l}}\left(z_{i}(k), z_{j}(k)\right)$ is a bilinear or fractional function.

As we mentioned before, a polynomial non-convex term in the objective function or in the constraints can be recursively expressed as an equivalent system of bilinear equations. As a result, in principle, the proposed convexified opti$\mathrm{mal} /$ model predictive control approaches can be used for all systems with nonconvex polynomial expressions.

\subsection{Recap of Dynamic Programming}

Dynamic programming is a closed-loop, global optimal control method (global optimal up to approximations due to state-input gridding and interpolations). It is based on the "principle of optimality" [16] which simply says that in a multi-stage process whatever the previous states are, the remaining decisions must be optimal with regard to the state following from the current state. This principle allows the optimal control problem of a $K$-stage process to be recursively formulated starting from the last stage. If $f \triangleq f_{c}(x(k), u(k), w(k))+f_{n c_{0}}\left(z_{1}(k), z_{2}(k)\right) g_{0}(w(k))$ denotes the function inside the summation of the cost function $J$ in Eq.(1), then

$$
\begin{aligned}
J_{N-K, N}^{\star}(x(N-K))= & \min _{u(N-K)}\{f(x(N-K), u(N-K), w(N-K))\}+ \\
& J_{N-(K-1), N}^{\star}(x(N-K), u(N-K), w(N-K)), \quad(13)
\end{aligned}
$$

where $J_{N-K, N}^{\star}$ is the optimal cost of the $K$-stage policy starting from stage $N-K$ to the final stage $N$ and $J_{N-(K-1), N}^{\star}$ is the optimal cost of the $K-1$-stage policy. To start the algorithm, we set $J_{N, N}^{\star}=0$, which is the cost of stage number zero, $K=0$. For dynamic programming-based control methods, the most important issue is to have an accurate model with minimum number of states and inputs due to the famous curse of dimensionality problem $[16,17]$. The reader is referred to references $[16,17]$ for details on dynamic programming.

\section{PART II: Applications}

The purpose of this section is to test the proposed convexification-based control methods first on a benchmark HVAC building control system case study from the literature to which we apply the convexified MPC control method and compare these control results with the adaptive and fuzzy control results from the literature. 
Next, the optimal control of a HybGCHP system is considered, which is the main application example for the developed methods. For the HybGCHP system, the convexified optimal control results are compared with the dynamic programming control results of the same system to assess the performance of the convexified optimal control method.

\subsection{Application to a Benchmark Problem}

The benchmark case study considered in this paper is the building heating control system, as described by Calvino et al. [18] and Chaudhry and Das [19]. The building heating system has not been described in detail, but is represented by a simplified dynamic model [18]. Starting from the instantaneous energy balance of the building heating system and some rough assumptions (which we copy here to allow comparison): (a) the energy balance accounts for thermal energy storage, thermal losses to the outside, internal gains and heat supplied by the heatdistributing devices, (b) only transmission losses are accounted for, no ventilation losses, (c) only indoor air capacity is included in the storage term (particular interest in fast variations of indoor air temperature), (d) correlation used for the thermal power of the heat-distributing device in non-nominal conditions (in this correlation the exponent of the system is set to 1 for computational convenience, imitating convection without enclosure), the following equation representing the dynamic behaviour of the building heating system is derived:

$$
\frac{d T_{i}(t)}{d t}=A u(t)\left(T_{f a v}-T_{i}(t)\right)^{\alpha}+B\left(T_{a}-T_{i}(t)\right)+C
$$

where

$$
\begin{aligned}
& u=\dot{m}, \quad A=c_{f}\left(\frac{T_{f, \text { in }}-T_{f, \text { out }}}{C_{a} \Delta T_{n}^{\alpha}}\right)=c_{f} \frac{\Delta T_{f}}{C_{a} \Delta T_{n}^{\alpha}}, \quad B=\frac{H_{T}}{C_{a}}, \quad C=\frac{\dot{Q}_{\text {gain }}}{C_{a}}, \\
& T_{\text {fav }}=\frac{T_{f, \text { in }}+T_{f, \text { out }}}{2}
\end{aligned}
$$

where $T_{i}$ is the indoor air temperature, $\dot{m}$ is the mass flow rate of the heating device, $T_{f, \text { in }}, T_{f, \text { out }}$ are the inlet and outlet temperatures of the heat carrier, respectively, $\alpha$ is the constant exponent characterizing the heat emission system, $T_{a}$ is the outdoor air temperature, $c_{f}$ is the specific heat capacity of the heat carrier, $C_{a}=c_{a} \rho_{a} V_{a}$ is the indoor air capacity, $\Delta T_{n}$ is the difference between the average heat carrier temperature and the indoor air temperature in nominal test conditions, 
$H_{t}$ is the global heat transfer coefficient of the building envelope, $\dot{Q}_{\text {gain }}$ represents the thermal power associated to internal gains. The model parameter values used in Eq.(14) are taken from Chaudhry and Das [19] and are given in Table 1. The variation of outside temperature is taken as $T_{a}=5+0.75 \sin (2 \pi \times 0.00003 t)$, where $t$ is the time in seconds.

Figures 1-2 show results from [19] when an adaptive controller and a fuzzy controller are used for the discretized version of Eq.(14), respectively. Figure 3 shows the corresponding results when convexified MPC is used with $N_{p}=10$. The system is controlled over $24 \mathrm{~h}$. The mass flow rate of the heating device is the control input and limited to maximum $0.1 \mathrm{~kg} / \mathrm{s}$. The total energy use of adaptive, fuzzy and convexified MPC controllers is $1.94 \times 10^{8} \mathrm{~J}, 2.01 \times 10^{8} \mathrm{~J}$ and $1.93 \times 10^{8} \mathrm{~J}$, respectively. From the figures and these results, we see that the tracking performance of convexified-MPC is better than the fuzzy controller and as good as the adaptive controller and the convexified MPC controller outperforms the adaptive and fuzzy controllers in terms of the amount of consumed energy.

Table 1: Model parameters of the simulated building HVAC system [19]

\begin{tabular}{|l|l|}
\hline$C_{a}=330.2 \mathrm{~kJ} / \mathrm{K}$ & $c_{f}=4186 \mathrm{~J} / \mathrm{kgK}$ \\
\hline$T_{f, \text { in }}=75^{\circ} \mathrm{C}$ & $\dot{Q}_{\text {gain }}=100 \mathrm{~W}$ \\
\hline$T_{f, \text { out }}=65^{\circ} \mathrm{C}$ & $T_{\text {fav }}=70^{\circ} \mathrm{C}$ \\
\hline$H_{T}=183 \mathrm{~W} / \mathrm{K}$ & $\Delta T_{f}=10^{\circ} \mathrm{C}$ \\
\hline$\alpha=1$ & $\Delta T_{n}=50^{\circ} \mathrm{C}$ \\
\hline
\end{tabular}

\subsection{Application to a HybGCHP System}

\subsubsection{HybGCHP System Description}

The HybGCHP system is presented in Figure 4 and consists of a heat pump, a gas-fired boiler, a passive cooler and a chiller. It is assumed that the heat demand $\left(\dot{Q}_{h}\right)$ is provided by the heat pump and the boiler and the cold demand $\left(\dot{Q}_{c}\right)$ is provided by the passive cooler (using a heat exchanger instead of an active chiller) and the chiller. The expressions for the efficiency $(\eta)$ and coefficients of performance (COP) of all components presented in Figure 4 are given by

$$
C O P_{h p}=\frac{\dot{Q}_{h p}}{P_{h p}}, \eta_{g b}=\frac{\dot{Q}_{g b}}{P_{g b}}, C O P_{p c}=\frac{\dot{Q}_{p c}}{P_{p c}}, C O P_{c h}=\frac{\dot{Q}_{c h}}{P_{c h}},
$$

where $P_{h p}$ denotes the electrical power consumption of the heat pump compressor and the power consumed by circulation pumps from the borefield side, $P_{g b}$ 


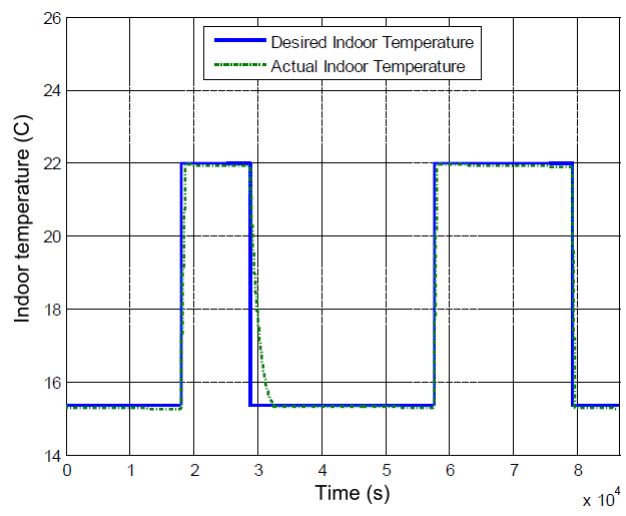

(a) $T_{i}-T_{\text {ref }}$

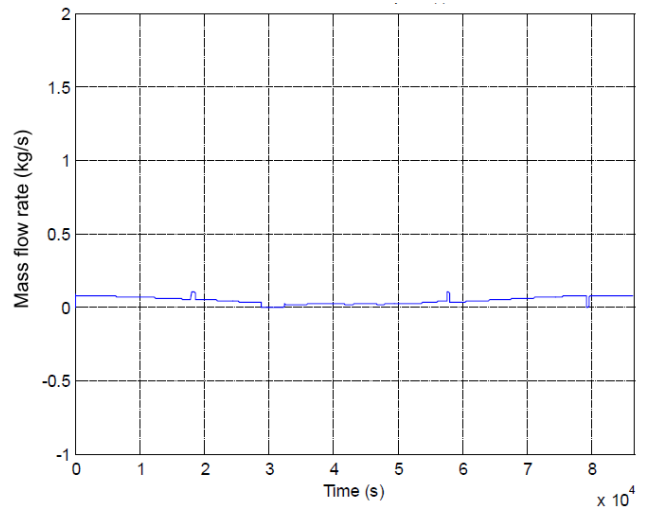

(b) $u$

Figure 1: Adaptive control: indoor temperature response and control input, figure taken from [19].

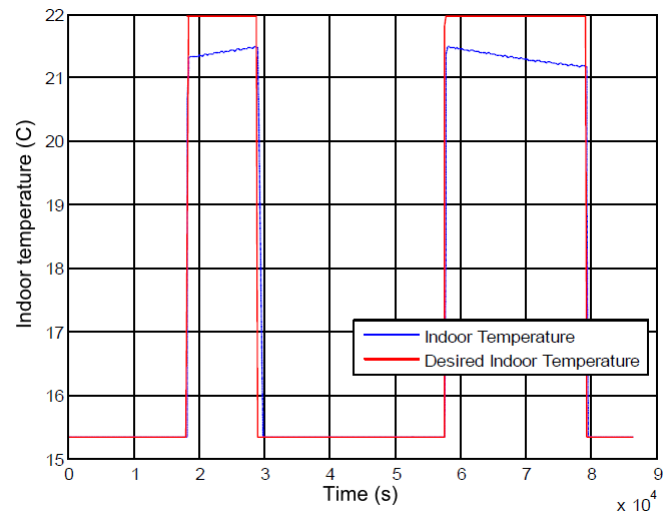

(a) $T_{i}-T_{\text {ref }}$

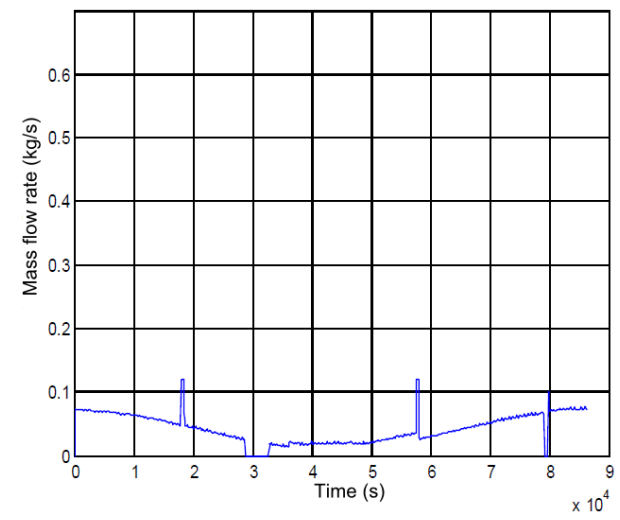

(b) $u$

Figure 2: Fuzzy control: indoor temperature response and control input, figure taken from [19].

denotes the power contained in the natural gas fed to the boiler, $P_{p c}$ the electricity power used for the circulation pumps of the passive cooling installation and finally $P_{c h}$ denotes the electricity power consumption of the chiller compressor and the circulation pumps of the cooling tower. The coefficients of performance given by the above expressions depend on the temperatures of the source and the emission 


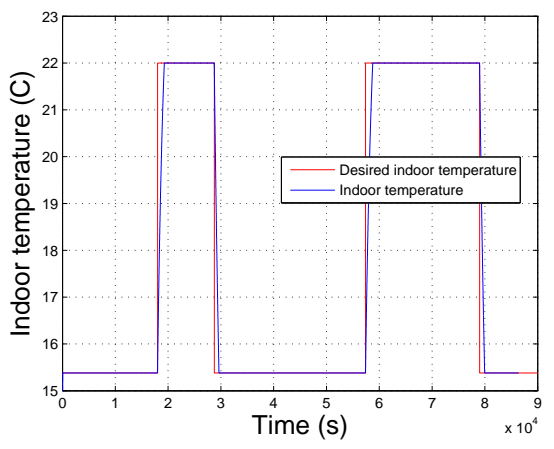

(a) $T_{i}-T_{\text {ref }}$

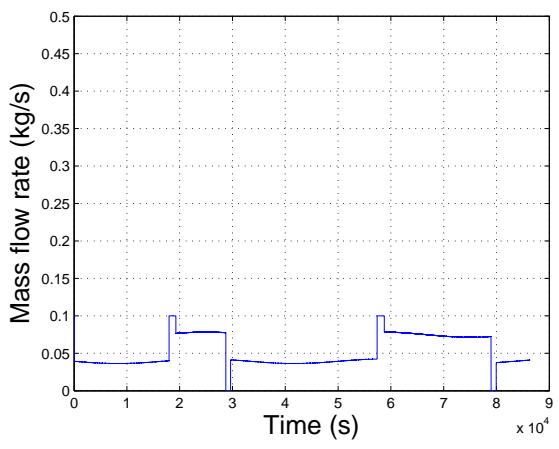

(b) $u$

Figure 3: Convexified MPC control: indoor temperature response and control input.

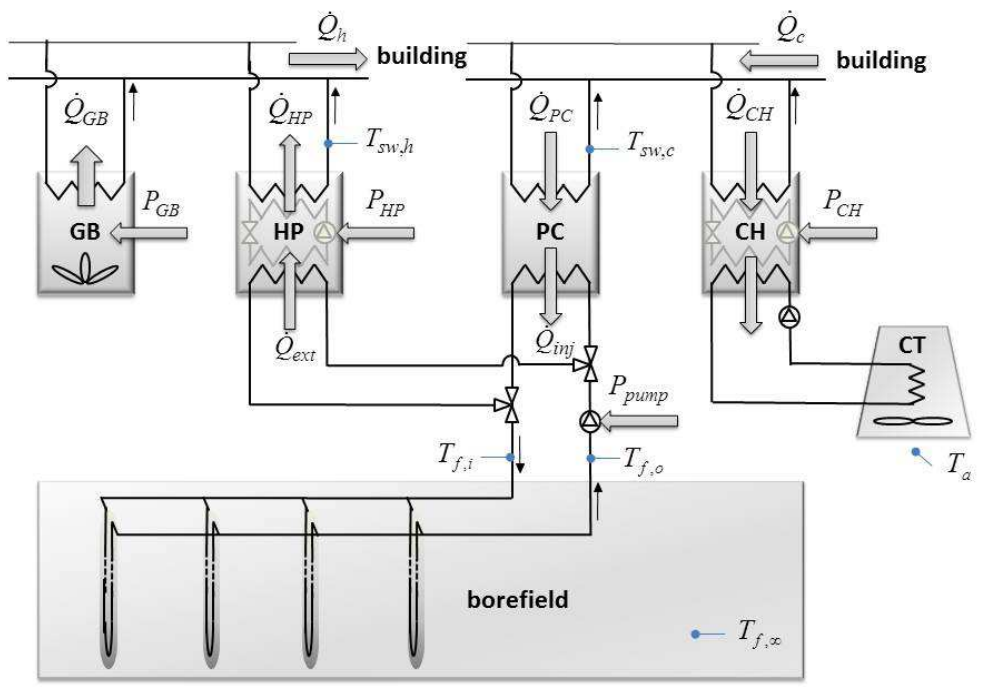

Figure 4: Schematic presentation of HybGCHP system: heat pump, gas-fired boiler, passive cooler and chiller.

system, as expressed by

$$
C O P_{h p}=f_{h p}\left(T_{f}, T_{s w, h}\right), C O P_{p c}=f_{p c}\left(T_{f}, T_{s w, c}\right), C O P_{c h}=f_{c h}\left(T_{a}, T_{s w, c}\right),
$$


where $T_{s w, h}, T_{s w, c}$ represent the supply water temperature for heating and supply water temperature for cooling, respectively. $T_{a}$ is the ambient temperature and $T_{f}$ is the borefield circulating fluid mean temperature, which is the average of inlet and outlet temperatures. The gas boiler efficiency, $\eta_{g b}$, is given a constant value of unity.

The electricity price $c_{e}(t)=0.15$ Euro/ $(\mathrm{kWh})$ for the day tariff, $c_{e}(t)=$ $0.09 \mathrm{Euro} /(\mathrm{kWh})$ for the night tariff and $c_{g}(t)$ is taken $0.06 \mathrm{Euro} /(\mathrm{kWh})[5]$. Here, COPs are fitted to TRNSYS data [20]:

$$
\begin{aligned}
C O P_{h p}(t) & =\alpha_{0}+\alpha_{1} T_{f}(t), \\
C O P_{c h}(t) & =\beta_{0}+\beta_{1} T_{a}(t)+\beta_{2} T_{a}^{2}(t), \\
C O P_{p c} & =12,
\end{aligned}
$$

where $C O P_{h p}$ and $C O P_{c h}$ are fitted for a supply water temperature of $T_{s w, h}=$ $40^{\circ} \mathrm{C}$ for heating and a supply water temperature of $T_{s w, c}=18^{\circ} \mathrm{C}$ for cooling.

Using $c_{e}(t)$ and $c_{g}(t)$, the objective function of the optimal control problem to be solved to minimize the energy-use cost function for the HybGCHP system operation over a time period $\left[t_{0}, t_{f}\right]$ is

$$
\begin{aligned}
J & =\int_{t_{0}}^{t_{f}}\left[c_{e}(t)\left(P_{h p}(t)+P_{p c}(t)+P_{c h}(t)\right)+c_{g}(t) P_{g b}(t)\right] d t \\
& =\int_{t_{0}}^{t_{f}}\left[c_{e}(t)\left(\frac{\dot{Q}_{h p}(t)}{C O P_{h p}(t)}+\frac{\dot{Q}_{p c}(t)}{C O P_{p c}}+\frac{\dot{Q}_{c h}(t)}{C O P_{c h}(t)}\right)+c_{g}(t) \frac{\dot{Q}_{g b}(t)}{\eta_{g b}}\right] d t \\
& \cong \sum_{k=0}^{N-1} t_{s}\left[c_{e}(k)\left(\frac{\dot{Q}_{h p}(k)}{C O P_{h p}(k)}+\frac{\dot{Q}_{p c}(k)}{C O P_{p c}}+\frac{\dot{Q}_{c h}(k)}{C O P_{c h}(k)}\right)+c_{g}(k) \frac{\dot{Q}_{g b}(k)}{\eta_{g b}}\right],
\end{aligned}
$$

where $t_{s}$ is the sampling period and " $\mathrm{k}$ " is the sampling instant. The cost function presented by Eq.(16) has to be minimized under operational temperature constraints, heat/cold demands, maximum net heat injected in the ground and power constraints. Next, we will discuss these constraints and present their expressions.

\subsubsection{Heat \& Cold Demand Satisfaction}

The building heating and cooling demands should be satisfied with some acceptable violation margins:

$$
\begin{gathered}
\dot{Q}_{h}(t)-\varepsilon_{h-l}(t) \leq \dot{Q}_{h p}(t)+\dot{Q}_{g b}(t) \leq \dot{Q}_{h}(t)+\varepsilon_{h-u}(t), \\
\dot{Q}_{c}(t)-\varepsilon_{c-l}(t) \leq \dot{Q}_{p c}(t)+\dot{Q}_{c h}(t) \leq \dot{Q}_{c}(t)+\varepsilon_{c-u}(t),
\end{gathered}
$$


where $\dot{Q}_{h}(t)$ and $\dot{Q}_{c}(t)$ are the building heat and cold demands, respectively, $\varepsilon_{h-l}(t), \varepsilon_{h-u}(t)$ are time-dependent lower and upper violation margins for heating demand and $\varepsilon_{c-l}(t), \varepsilon_{c-u}(t)$ the lower and upper violation margins for cooling demand. Note that the margins are taken to be time-dependent to allow different degrees of flexibility over time. During critical demand load periods these margins can be set very strictly. It is assumed that $\dot{Q}_{h}$ and $\dot{Q}_{c}$ are given and hence building modeling is not included in the optimization.

\subsubsection{Circulating Fluid Temperature Bounds}

The cooling of a building requires heat injection into the ground during summer. This increases the ground temperature towards winter, which, in turn, increases $\mathrm{COP}_{\mathrm{hp}}$. However, the ground temperature, which is represented indirectly by $T_{f}$, should be kept below the supply water temperature, $T_{s w, c}$, for passive cooling of the building. Similarly, heating of a building requires heat extraction from the ground. This decreases the ground temperature towards summer, which, in turn, increases $\mathrm{COP}_{\mathrm{pc}}$. However, again the ground temperature represented indirectly by $T_{f}$ should not decrease to a value below freezing point to avoid frost problems. All these aspects require to put lower and upper bounds on $T_{f}$ :

$$
T_{f-\min }(t)<T_{f}(t)<T_{f-\max }(t) .
$$

\subsubsection{Heat Exchange with Ground and Bounds on Annual Net Heat Transfer with Ground}

The heat extraction/injection power from/to the ground and the net heat transfer with the ground are given by the following equations

$$
\begin{aligned}
& \dot{Q}_{e x t}(t)=\frac{C O P_{h p}(t)-1}{C O P_{h p}(t)} \dot{Q}_{h p}(t), \\
& \dot{Q}_{i n j}(t)=\frac{C O P_{p c}+1}{C O P_{p c}} \dot{Q}_{p c}(t), \\
& \dot{Q}_{\text {net }}(t)=\dot{Q}_{i n j}(t)-\dot{Q}_{e x t}(t) .
\end{aligned}
$$

To limit the degree of thermal build up or depletion in the ground, the following bound may be put on the net annual heat transfer with the ground

$$
\left|\sum_{\text {1year }} \dot{Q}_{\text {net }}\right|<\dot{Q}_{\max } \text {. }
$$

Remark 1: If for some applications Eq.(20) is too stringent, then there are two options: (a) remove the constraint or (b) make $\dot{Q}_{\max }$ higher. 


\subsubsection{Borehole Dynamics}

In this section the borefield shown in Figure 4 is modeled as a single equivalent borehole which is sized according to the specified building loads to be considered in the next sections. This is an approximation neglecting the interaction between different boreholes in a borefield. The equivalent borehole filled with grout is schematically shown in Figure 5, where an equivalent diameter approach [21, 2, $22]$ is used. In the equivalent diameter approach, the heat transfer from the Utube is approximated by the heat transfer from a single pipe with a hypothetical diameter through which the heat exchanging fluid circulates.

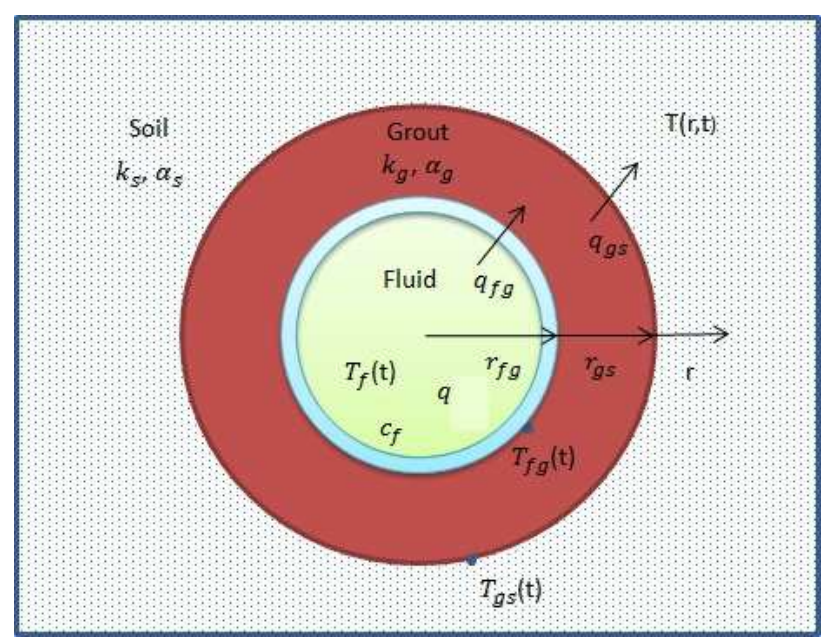

Figure 5: Schematic presentation of the equivalent-diameter borehole system.

The objective is the determination of the circulating fluid mean temperature, $T_{f}(t)$, corresponding to a net (injected - extracted) heat profile per unit length, $u_{\text {net }}=q$. The one-dimensional radial conduction heat transfer equation is considered. Next, the grout and soil regions are divided into thermal nodes and an energy balance for the equivalent borehole is considered. Using the finite volume technique, the following large-scale dynamic model is obtained

$$
\begin{aligned}
& x_{e}(k+1)=A_{e}(p) x_{e}(k)+B_{e}(p) u_{\text {net }}(k), \\
& y(k)=T_{f}(k)=C_{e} x_{e}(k),
\end{aligned}
$$

where $x_{e}=\left[\begin{array}{llll}T_{f} & T_{g_{1}} \cdots T_{g_{n g}} T_{s_{1}} \cdots T_{s_{n_{s}}}\end{array}\right]^{T}, A_{e}, B_{e}, C_{e}$ are matrices for the borehole dynamics. Here, $T_{f}$ is the circulating fluid mean temperature, $\left[T_{g_{1}} \cdots T_{g_{n_{g}}}\right]^{T}$ are grout nodal temperatures, $\left[T_{s_{1}} \cdots T_{s_{n_{s}}}\right]^{T}$ are soil (ground) nodal temperatures and $p$ is the known parameter vector including thermal, physical and other 
parameters of the system (diffusivities $\alpha_{g}, \alpha_{s}$, conductivities $k_{g}, k_{s}$, different radii $r_{f g}, r_{g s}$, discretization step sizes, etc.) and $u_{\text {net }}$ is the net heat power injected to the ground per borehole length. A full derivation of Eq.(21) can be found in [6].

The next step is to obtain a reduced-order model from the large-scale model given in Eq.(21) which has 506 states. Model order reduction is necessary to prevent computational difficulties in controller design and/or to have a fast controller. Even sometimes model order reduction is necessary. For example, largescale models are, in general, non-observable and for a closed-loop control design method with state estimation necessity, the controller model should be observable. As a model-order reduction technique, we use the Proper Orthogonal Decomposition (POD) method [23, 24]. POD is a very commonly used model order reduction technique in many engineering fields, especially in fluid mechanics, with real-time implementations [25, 26, 27].

The reduced-order model is given by

$$
\begin{aligned}
x_{r}(k+1) & =A_{r} x_{r}(k)+B_{r} u_{\text {net }}(k), \\
y(k) & =C_{r} x_{r}(k),
\end{aligned}
$$

and details can be found in [6]. The model order of the chosen reduced-order model is four. Figure 6 shows mean and maximum absolute mismatch error for $T_{f}$ of reduced-order models with respect to that of the large-scale model as a function of model order. The results are based on model simulations with a multi-sine input signal consisting of 30 sines. From Figure 6 we get a maximum absolute mismatch error for $T_{f}$ around $0.63^{\circ} \mathrm{C}$ and a mean absolute mismatch error around $0.14^{\circ} \mathrm{C}$ for a reduced-order model of fourth order.

Remark 2: POD is a flexible model-order reduction method compared to other model order reduction methods. In principle, other model order reduction methods can also be used for the considered system. One of the main features of POD is that it can be applied both to linear and nonlinear ODEs. Another flexibility in POD is the ability to reflect both short and long term effects in the reduced-order model by adjustment of the time instants in the construction of snapshot matrix.

Remark 3: The only constraint for a successful POD-based reduced-order modeling is that the large-scale model of the underlying system should be accurate. There is no limit on the level of detail. The large-scale model can even be a nonlinear model such as the Navier-Stokes equation. If parameter tuning is needed, these parameters can be tuned through parameter estimation methods in partial 

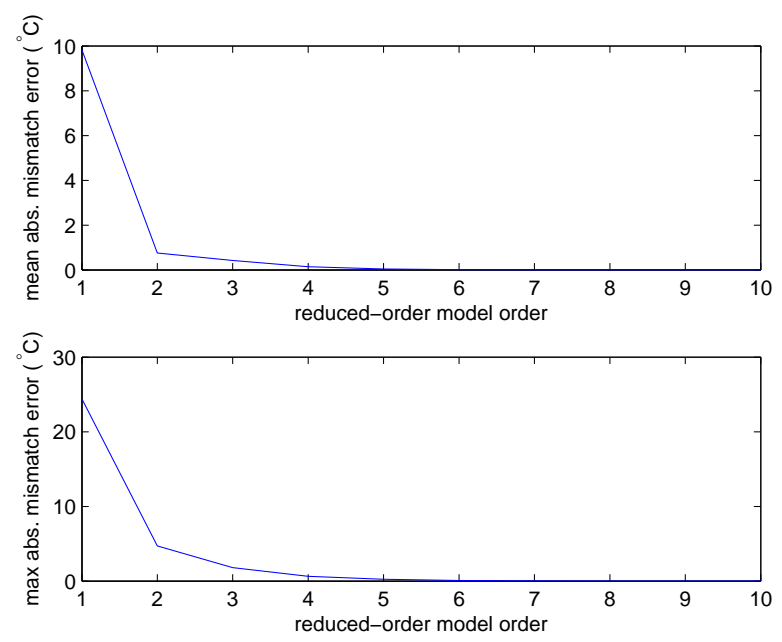

Figure 6: Reduced-order model $T_{f}$ mismatch error as a function of model order.

differential equations before application of POD [28]. Alternatively, if the underlying large-scale model includes some parameters to be tuned using real measurements, then there are some model reduction techniques collectively called PROM (parameterized reduced-order modeling) where a parameterized reducedorder model is obtained with the same parameters as the large-scale model [29]. In such a case, instead of the large scale model, the parameterized reduced-order model can be used for parameter tuning using real measurements.

Remark 4: The developed reduced-order model obtained from a large-scale model definitely has the potential to be better than simple models for optimal control purposes. The main reason for this is as follows. Here, we consider optimal control which is an open-loop control method where control actions are calculated off-line and the accuracy of these control actions is depending on the "simulation performance" of the model, not on its "prediction performance" because in classical optimal control measured values are not used and everything is done off-line. A too simple model cannot have a good simulation performance over a long period (like one year or multiple years), as shown in Figure 6. If the control method is MPC or dynamic programming, then simple models may have a good prediction performance (which uses past measured outputs). In such cases, simple models may be preferred over complex models. However, in classical open-loop optimal control they typically fail. 


\subsubsection{Non-convex Optimal Control Problem for Total Energy Cost Minimization}

Letting $u_{1} \triangleq \dot{Q}_{h p}, u_{2} \triangleq \dot{Q}_{g b}, u_{3} \triangleq \dot{Q}_{c h}, u_{4} \triangleq \dot{Q}_{p c}, \tilde{u}_{1} \triangleq \frac{\dot{Q}_{h p}}{C O P_{h p}}, u_{n e t} \triangleq$ $\dot{Q}_{n e t}, C O P_{p c}=12, \eta_{g b}=1$, we obtain the following optimization problem with the associated constraints from the related equations defined in Section 3.2.1:

$\operatorname{minimize} \sum_{k=0}^{N-1} t_{s}\left[c_{e}(k)\left(\tilde{u}_{1}(k)+\frac{u_{4}(k)}{12}+\frac{u_{3}(k)}{\beta_{0}+\beta_{1} T_{a}(k)+\beta_{2} T_{a}^{2}(k)}\right)+c_{g}(k) u_{2}(k)\right]$,

subject to

$\dot{Q}_{h}(k)-\varepsilon_{h-l}(k) \leq u_{1}(k)+u_{2}(k) \leq \dot{Q}_{h}(k)+\varepsilon_{h-u}(k) \quad$ (heating demand satisfaction),

$\dot{Q}_{c}(k)-\varepsilon_{c-l}(k) \leq u_{3}(k)+u_{4}(k) \leq \dot{Q}_{c}(k)+\varepsilon_{c-u}(k) \quad$ (cooling demand satisfaction),

$u_{\text {net }}(k)=\frac{13}{12} u_{4}(k)-u_{1}(k)+\tilde{u}_{1}(k) \quad\left(\right.$ expression for $\left.u_{\text {net }}(k)\right)$,

$x_{r}(k+1)=A_{r} x_{r}(k)+B_{r} u_{n e t}(k) \quad$ (borehole state-space dynamics),

$T_{f}(k)=C_{r} x_{r}(k) \quad$ (output equation),

$T_{f-\min }<T_{f}(k)=C_{r} x_{r}(k)<T_{f-\max } \quad$ (circulating fluid temperature bounds),

$\left|\sum_{k=0}^{N-1} u_{\text {net }}(k)\right|<\dot{Q}_{\max } \quad$ (bounding the total net heat injected to the ground),

$C O P_{h p}(k)=\alpha_{0}+\alpha_{1} C_{r} x_{r}(k) \quad$ (heat pump coefficient of performance),

$u_{1}(k)=C O P_{h p}(k) \tilde{u}_{1}(k)$ (relation between $u_{1}, C O P_{h p}$ and $\left.\tilde{u}_{1}\right)$.

With this formulation, the non-convexity of the optimal control problem comes from Eq.(23j): a bilinear term. Note that the rational term including $T_{a}$ in the cost function does not create any non-convexity because $T_{a}$ is not a decision variable or a function of decision variables. In the next subsection, Eq.(23j) will be replaced by its convex approximation. 


\subsubsection{Convexified Optimal Control Problem and Comparison with Dynamic Pro- gramming}

The convexified optimal control problem is the same problem as the one presented by Eq.(23) except Eq.(23j) is replaced by Eq.(10) with

$$
w=u_{1}(k), \quad x_{1}=C O P_{h p}, \quad x_{2}=\tilde{u}_{1}(k) .
$$

Note that all other constraints are already/or can be easily put into the form given in Eq.(7). The lower bound for the coefficient of performance of the heat pump, $C O P_{h p}$, is set to 2 and the upper bound, $\overline{C O P_{h p}}$, is set to 6. Similarly, the lower bound of $\tilde{u}_{1}=\dot{Q}_{h p} / C O P_{h p}, \underline{\tilde{u}_{1}}$, is set to $0 \mathrm{~W}$ and the upper bound, $\overline{\tilde{u}}_{1}$, is set to $3000 \mathrm{~W}$, assuming a maximum heat pump power of $6000 \mathrm{~W}$. The convexified optimal control problem is coded using YALMIP [30] in MATLAB and calling CPLEX [31] as solver. Note that in convex optimization problems, any local minimum is also a global minimum. This nice property makes convex optimization formulations very attractive.

It is important to know that optimal control is an open-loop control method and hence a successful control application in real-time requires the controller model to be very accurate and full access to other inputs affecting the system. However, often, the aim of optimal control is to see the maximum potential or to assess the performance of closed-loop control methods.

To apply dynamic programming for assessing the performance of convexified optimal control, the optimization problem given by Eq.(23) is equivalently reformulated with two inputs instead of four by assuming zero violation margins (see Eq.(17)) to alleviate the curse of dimensionality problem in dynamic programming. The requirements of heating and cooling load satisfaction give (ignoring violation flexibilities)

$$
\begin{gathered}
\dot{Q}_{g b}(k)=\dot{Q}_{h}(k)-\dot{Q}_{h p}(k), \\
\dot{Q}_{p c}(k)=\dot{Q}_{c}(k)-\dot{Q}_{c h}(k) .
\end{gathered}
$$

Then, the expression inside the summation in the cost function (16) becomes

$$
\begin{gathered}
f\left(\dot{Q}_{h p}, \dot{Q}_{c h}, \dot{Q}_{h}, \dot{Q}_{c}, C O P_{h p}, C O P_{c h}, c_{e}, c_{g}\right)=t_{s}\left[\frac{c_{e}(k)}{C O P_{h p}(k)}-\frac{c_{g}(k)}{\eta_{g b}}\right] \dot{Q}_{h p}(k)+ \\
t_{s}\left[\frac{c_{e}(k)}{C O P_{c h}(k)}-\frac{c_{e}(k)}{C O P_{p c}}\right] \dot{Q}_{c h}(k)+t_{s}\left[\frac{c_{g}(k)}{\eta_{g b}} \dot{Q}_{h}+\frac{c_{e}(k)}{C O P_{p c}} \dot{Q}_{c}\right]
\end{gathered}
$$


To put a constraint on $y=T_{f}=C_{r} x_{r}$, we prefer to transform the borehole state dynamics in reduced-states as $\tilde{x}_{r}=T x_{r}$, where

$$
T=\left(\begin{array}{cc}
C_{r} & \\
0_{3 \times 1} & I_{3}
\end{array}\right)
$$

such that $\tilde{x}_{r_{1}}=C_{r} x_{r}=y=T_{f}$. The reason for such transformation is the fact that this formulation allows an easy use of an existing dynamic programming toolbox [32]. Then, the reduced-order transformed borehole state-space dynamics becomes

$$
\begin{aligned}
\tilde{x}_{r}(k+1) & =\tilde{A}_{r} \tilde{x}_{r}(k)+\tilde{B}_{r} u_{\text {net }}(k), \\
y(k) & =T_{f}(k)=\tilde{x}_{r_{1}}(k),
\end{aligned}
$$

so that the first state becomes output and hence the circulating fluid temperature bounds can be put on the first state. The optimization problem to be solved by dynamic programming is as follows:

$$
\operatorname{minimize} \sum_{k=0}^{N-1} f\left(\dot{Q}_{h p}, \dot{Q}_{c h}, \dot{Q}_{h}, \dot{Q}_{c}, C O P_{h p}, C O P_{c h}, c_{e}, c_{g}\right)
$$

subject to

$\tilde{x}_{r}(k+1)=\tilde{A}_{r} \tilde{x}_{r}(k)+\tilde{B}_{r} u_{\text {net }}(k) \quad$ (borehole state-space dynamics),

$T_{f-\min }<T_{f}(k)=\tilde{x}_{r_{1}}(k)<T_{f-\max } \quad$ (circulating fluid temperature bounds),

$\left|\sum_{k=0}^{N-1} u_{\text {net }}(k)\right|<\dot{Q}_{\max } \quad$ (bounding the total net heat injected to the ground),

$C O P_{h p}(k)=\alpha_{0}+\alpha_{1} \tilde{x}_{r_{1}}(k) \quad$ (heat pump coefficient of performance),

$u_{n e t}(k)=\frac{13}{12}\left[\dot{Q}_{c}(k)-\dot{Q}_{c h}(k)\right]-\frac{C O P_{h p}(k)-1}{C O P_{h p}(k)} \dot{Q}_{h p}(k) \quad\left(\right.$ expression for $\left.u_{n e t}(k)\right)$.

Note that for $f(x, u, w)$ in Eq.(25), we have $u=\left[\dot{Q}_{h p} \dot{Q}_{c h}\right]^{T}, w=\left[\dot{Q}_{h}, \dot{Q}_{c}, C O P_{h p}\right.$, $\left.C O P_{c h}, c_{e}, c_{g}\right]^{T}$ and $C O P_{\mathrm{hp}}$ depends on the state $\tilde{x}_{r_{1}}$. Dynamic programming is applied to (28). Typically, in dynamic programming the feasible states and feasible inputs are gridded into quantized values and then at a given stage with a 
chosen feasible state value, all possible quantized inputs are tried until the minimum of (28a) is determined at that stage. This is done for all feasible gridded states. Hence, the dynamic programming control algorithm is a closed-loop and global optimal control algorithm (global optimal up to the approximations due to state-input gridding and interpolations). As mentioned before, for dynamic programming-based control methods, the most important issue is to have an accurate model with minimum number of states/inputs due to the curse of dimensionality problem. The nonlinearity of the model is not an issue for dynamic programming. Interpolation is required when the measured/estimated states are not the gridded states during controller implementation. For application of dynamic programming, the control input range for both $\dot{Q}_{h p}$ and $\dot{Q}_{c h},[0,6000] W$, was gridded into 60 points. All other transformed states of the reduced-order model were divided into 50 grid points. The lower and upper circulating fluid mean temperature bounds were taken to be $0.5^{\circ} \mathrm{C}$ and $15.5^{\circ} \mathrm{C}$, respectively, and $\dot{Q}_{\max }=1000 \mathrm{~W}$.

The comparison of control results for convexified optimal control and dynamic programming based optimal control is shown in Figures 7-8. Simulations are run for a duration of one year and with a control time step of 4 hours. Figures 7(a)-7(b) show the time evolution of the mean temperature of the circulating fluid $\left(T_{f}\right)$, Figures 7(c)-7(d) show the heat pump power and heating demand $\left(\dot{Q}_{h p}, \dot{Q}_{h}\right)$, Figures $8(\mathrm{a})-8(\mathrm{~b})$ show the chiller power and cold demand $\left(\dot{Q}_{c h}, \dot{Q}_{c}\right)$ and finally Figures 8(c)-8(d) show the evolution of accumulated cost $(J)$.

From the comparison results we see that (a) the time evolution of variables are, in general, very close to each other (b) for the convexified optimal control case the circulating fluid is more oscillatory around the upper temperature bound, which is due to the more oscillatory action of the chiller (c) the circulating fluid mean temperature in the convexified optimal control case does not approach to the lower bound of $0.5^{\circ} \mathrm{C}$, which is due to the fact that in the convexified optimal control case $\dot{Q}_{h p}$ is a little bit lower than the corresponding $\dot{Q}_{h p}$ in the dynamic programming case during the initial control period (d) the annual cost of the system with convexified optimal control is only $3 \%$ higher than the corresponding value with dynamic programming control.

\subsection{HybGCHP System Control Using Convexified MPC}

The non-convex model predictive control (NMPC) problem for HybGCHP system is shortly described as follows. Given the borehole dynamics

$$
\begin{aligned}
x_{r}(k+1) & =A_{r} x_{r}(k)+B_{r} u_{\text {net }}(k), \\
y(k) & =C_{r} x_{r}(k),
\end{aligned}
$$




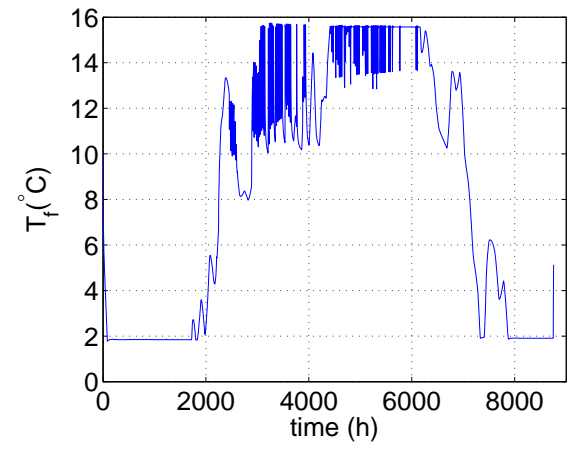

(a) Circulating fluid mean temperature (convex-OC).

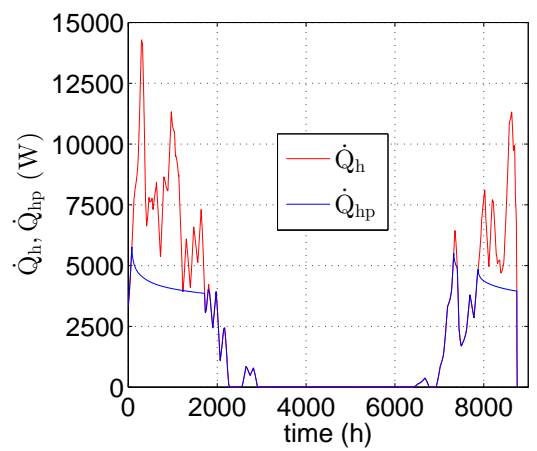

(c) Heat demand and heat pump power (convex-OC).

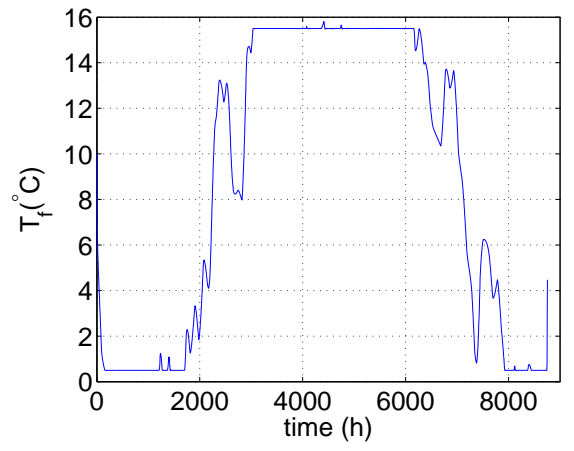

(b) Circulating fluid mean temperature (DP)

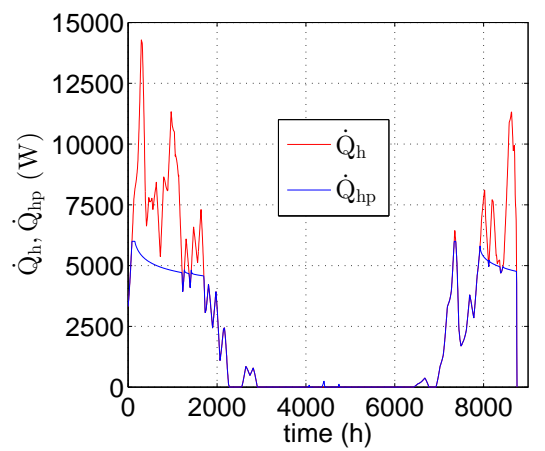

(d) Heat demand and heat pump power (DP).

Figure 7: Comparison of controlled system variables for convexified optimal control and dynamic programming.

the objective of NMPC is the minimization of

$$
\sum_{i=k}^{k+N_{p}} t_{s}\left[c_{e}(i)\left(\tilde{u}_{1}(i)+\frac{u_{4}(i)}{12}+\frac{u_{3}(i)}{\beta_{0}+\beta_{1} T_{a}(i)+\beta_{2} T_{a}^{2}(i)}\right)+c_{g}(i) u_{2}(i)\right]
$$




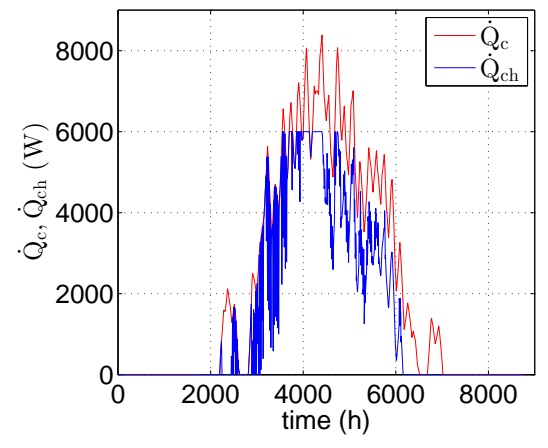

(a) Cold demand and chiller power (convex$\mathrm{OC})$.

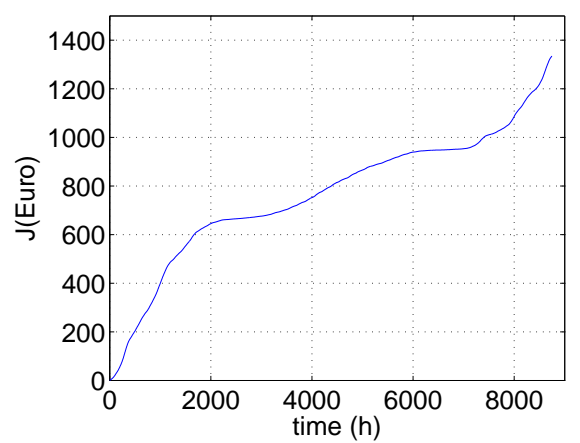

(c) Accumulated cost profile (convex-OC).

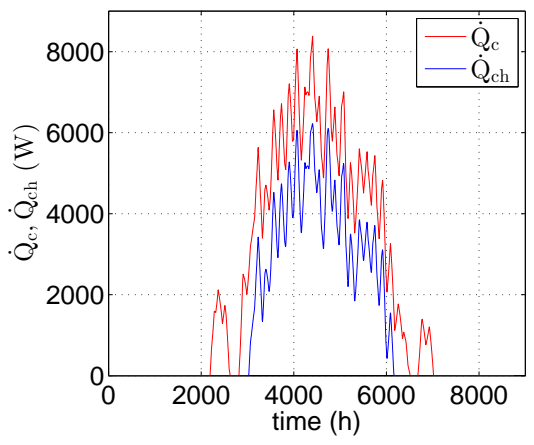

(b) Cold demand and chiller power (DP)

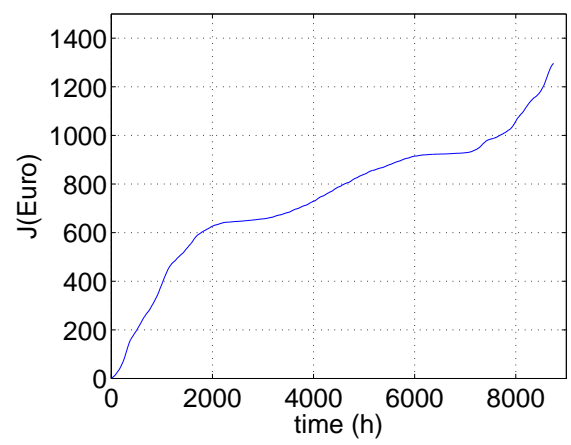

(d) Accumulated cost profile (DP).

Figure 8: Comparison of controlled system variables for convexified optimal control and dynamic programming.

subject to the constraints

$\dot{Q}_{h}(k)-\varepsilon_{h-l}(k) \leq u_{1}(k)+u_{2}(k) \leq \dot{Q}_{h}(k)+\varepsilon_{h-u}(k) \quad$ (heating demand satisfaction),

$\dot{Q}_{c}(k)-\varepsilon_{c-l}(k) \leq u_{3}(k)+u_{4}(k) \leq \dot{Q}_{c}(k)+\varepsilon_{c-u}(k) \quad$ (cooling demand satisfaction),

$u_{\text {net }}(k)=\frac{13}{12} u_{4}(k)-u_{1}(k)+\tilde{u}_{1}(k) \quad\left(\right.$ expression for $\left.u_{\text {net }}(k)\right)$,

$T_{f-\min }<T_{f}(k)=C_{r} x_{r}(k)<T_{f-\max } \quad$ (circulating fluid temperature bounds),

$\left|\sum_{k=0}^{N-1} u_{\text {net }}(k)\right|<\dot{Q}_{\max }$ (bounding the total net heat to the ground),

$C O P_{h p}(k)=\alpha_{0}+\alpha_{1} C_{r} x_{r} \quad$ (heat pump coefficient of performance),

$u_{1}(k)=C O P_{h p}(k) \tilde{u}_{1}(k) \quad$ (relation between $u_{1}, C O P_{h p}$ and $\left.\tilde{u}_{1}\right)$, 
where

$$
x_{r}(k+1)=A_{r} x_{r}(k)+B_{r} u_{\text {net }} \quad \text { with } \quad T_{f}(k)=C_{r} x_{r}(k) .
$$

and

$$
\begin{aligned}
& u_{1}(s)=u_{1}\left(k+N_{c}\right), \quad k+N_{c} \leq s \leq k+N_{p}, \quad N_{c} \leq N_{p}, \\
& u_{2}(s)=u_{2}\left(k+N_{c}\right), \quad k+N_{c} \leq s \leq k+N_{p}, \quad N_{c} \leq N_{p}, \\
& u_{3}(s)=u_{3}\left(k+N_{c}\right), \quad k+N_{c} \leq s \leq k+N_{p}, \quad N_{c} \leq N_{p}, \\
& u_{4}(s)=u_{4}\left(k+N_{c}\right), \quad k+N_{c} \leq s \leq k+N_{p}, \quad N_{c} \leq N_{p}, \\
& u_{\text {net }}(s)=u_{\text {net }}\left(k+N_{c}\right), \quad k+N_{c} \leq s \leq k+N_{p}, \quad N_{c} \leq t_{p} .
\end{aligned}
$$

Here $N_{p}$ and $N_{c}$ are called prediction and control horizons, respectively. Eq.(31e) is eliminated since this constraint cannot be applied in NMPC because NMPC accounts for only part of the future. The NMPC problem is in a fact a kind of optimal control problem over a shorter horizon. As a result, for the application of the proposed convexification to NMPC, the convexification of Eq.(31g) is performed, after which the NMPC becomes convex model predictive control. Here, we do not present the results for convexified MPC, we just wanted to show how the proposed idea can be used in the context of NMPC. It is not useful to compare convexified MPC results with dynamic programming since convexified MPC does not see the whole control period over which the system is controlled and this leads to different results.

Remark 5: In theory, NMPC or convex MPC can deal with a large number of states (like 506 states here) and hence it may seem that the large-scale model can be used directly. However, in practice this will cause the following problems. Firstly, the large-scale model with 506 states is not observable since there are 506 states and only one input-output pair. As a result, it is not possible to design a Kalman filter or any other estimator to be used in NMPC or convex MPC. Second, the use of a large-scale model in NMPC may create numerical and convergence problems. Finally, the computation of control inputs in NMPC or convex MPC with a large-scale model of 506 states will take a longer computation time.

\section{Conclusion}

In this paper a convexification approach using convex envelopes for hard-tosolve non-convex optimization problems involving rational and/or bilinear terms 
of decision variables was proposed. Such optimization problems may be encountered in optimal design or optimal/model predictive control of building HVAC systems. A general convex relaxation framework for such class of problems (non-convex optimal control and non-convex model predictive control problems) was proposed. Furthermore, the performance of convexified control methods was demonstrated on two applications: a simplified building HVAC system and a HybGCHP system.

For the first case study of an HVAC building control system, the performance of convexified MPC was compared to the performance of fuzzy and adaptive control from the literature. Convexified MPC outperformed adaptive and fuzzy controllers. For the second application, the total energy cost minimization of buildings with a HybGCHP system, convexified optimal control was used and its results were compared to dynamic programming based control results, which is a closedloop, global optimal control method (global optimal up to approximations in the gridding of states/inputs and used interpolations). The results of convex optimal control were close to (in terms of dynamic trends, global cost) these of dynamic programming, which validates again the effectiveness of the proposed method.

To the authors' best knowledge, the proposed method is the first in the field of optimization or control of building HVAC systems in energy efficient buildings. The overall message of this paper is that given a non-convex optimization/control problem of thermal systems, the first step should be to analyse the given system in terms of the non-convex terms and then investigate whether convex envelopes for the associated terms exist or not. If they exist, then these terms should be replaced with their convex approximations, thus approximating the original nonconvex optimization/control problem by a convex one. Note, however, that analytical convex approximations exist only for a set of non-convex functions and the numerical computation of the convex envelope for a general non-convex function may be computationally very difficult. Last but not least, in general convexified optimization results are better than linearized optimization results but are not as good as global optimal results. The accuracy degree of the approximation of the original non-convex optimization problem by a convex one may be very case dependent.

\section{Acknowledgements}

The authors want to acknowledge the Agency for Innovation by Science and Technology in Flanders (IWT) and the Scientific and Technical Center for the 
Building Industry (WTCB) for supporting the post-doctoral research work of Ercan Atam within the VIS SMART GEOTHERM project.

\section{References}

[1] M. LeBreux, M. Lacroix, G. Lachiver, Fuzzy and feedforward control of an hybrid thermal energy storage system, Energy and Buildings 38 (10) (2006) 1149-1155.

[2] A. Chiasson, Simulation and design of hybrid geothermal heat pump systems, Ph.D. thesis, University of Wyoming, USA (2007).

[3] J. Cullin, J. Spitler, Comparison of simulation-based design procedures for hybrid ground source heat pump systems, in: Proceedings of the 8th International Conference on System Simulation in Buildings, Liege, Belgium, 2010.

[4] S. Hackel, A. Pertzborn, Effective design and operation of hybrid groundsource heat pumps: Three case studies, Energy and Buildings 43 (12) (2011) 3497-3504.

[5] C. Verhelst, Model predictive control of hybrid ground-coupled heat pump systems in office building, Ph.D. thesis, Katholieke Universiteit Leuven, Belgium (2012).

[6] E. Atam, C. Verhelst, L. Helsen, Development of a control-oriented model for borehole dynamics for buildings equipped with ground-coupled heat pumps, in: BS 2013 Conference, Chambery, France, 2013.

[7] M. Esen, T. Yuksel, Experimental evaluation of using various renewable energy sources for heating a greenhouse, Energy and Buildings 65 (2013) 340351.

[8] A. Balbay, M. Esen, Experimental investigation of using ground source heat pump system for snow melting on pavements and bridge decks, Scientific Research and Essays 5 (24) (2010) 3955-3966.

[9] H. Esen, M. Inalli, A. Sengur, M. Esen, Modelling a ground-coupled heat pump system using adaptive neuro-fuzzy inference systems, International Journal of Refrigeration 31 (1) (2008) 65-74. 
[10] H. Esen, M. Inalli, A. Sengur, M. Esen, Forecasting of a ground-coupled heat pump performance using neural networks with statistical data weighting preprocessing, International Journal of Thermal Sciences 47 (4) (2008) 431441.

[11] District heating cooling - dhc, Online: http : //www.dhcplus.eu/wp content/uploads/2012/05/120529 - Vision - DHC - final.pdf (2014).

[12] F. De Ridder, M. Diehl, G. Mulder, J. Desmedt, J. Van Bael, An optimal control algorithm for borehole thermal energy storage systems, Energy and Buildings 43 (10) (2011) 2918-2925.

[13] S. Boyd, L. Vandenberghe, Convex Optimization, Cambridge University Press, 2004.

[14] G. McCormick, Mathematical programming computability of global solutions to factorable nonconvex programs: Part i- convex underestimating problems, Mathematical programming 10 (1976) 147-175.

[15] M. Locatelli, F. Schoen, Global Optimization: Theory, Algorithms, and Application, Mos-Siam Series on Optimization, 2013.

[16] D. Kirk, Optimal Control Theory, An introduction, Englewood Cliffs, New Jersey, 1970.

[17] D. Bertsekas, Dynamic Programming and Optimal Control, 2nd ed. Athena Scientific, 2000.

[18] F. Calvino, M. L. Gennusa, M. Morale, G. Rizzo, G. Scaccianoce, Comparing different control strategies for indoor thermal comfort aimed at the evaluation of the energy cost quality of building, Applied Thermal Engineering 30 (16) (2010) 2386-2395.

[19] I. S. Chaudhry, M. Das, A stable energy saving adaptive control scheme for building heating and cooling systems, Journal of Power and Energy Engineering 2 (2014) 14-25.

[20] TRNSYS, A transient simulation program: Trnsys 16 reference manual (1998).

[21] S. Javed, Thermal modeling and evaluation of borehole heat transfer, Ph.D. thesis, Chalmers University of Technology, Sweden (2010). 
[22] Y. Gu, D. O Neal, An analytical solution to transient heat conduction in a composite region with a cylindrical heat source, Journal of Solar Energy Engineering 117 (8) (1995) 242-248.

[23] P. Holmes, J. Lumley, B. G., Turbulence, Coherence Structure, Dynamical Systems and Symmetry, Cambridge University Press, Cambridge, 1996.

[24] P. Astrid, Reduction of process simulation models: a proper orthogonal decomposition approach, Ph.D. thesis, Technische Universiteit Eindhoven, Netherlands (2004).

[25] H. T. Banks, R. C. H. Del Rosario, H. T. Tran, Proper orthogonal decomposition-based control of transverse beam vibrations: experimental implementation, IEEE Transactions on Control Systems Technology 10 (5) (2002) 717-726.

[26] B. Apacoglu, S. Aradag, CFD analysis and reduced order modeling of uncontrolled and controlled laminar flow over acircular cylinder, Engineering Applications of Computational Fluid Mechanics 5 (1) (2011) 67-82.

[27] R. Niu, M. Skliar, Identification of reduced-order thermal therapy models using thermal mr images: Theory and validation, IEEE Trans Med Imaging 31 (7) (2012) 1493-1504.

[28] X. Xun, J. Cao, B. Mallick, A. Maity, R. J. Carrol, Parameter estimation of partial differential equation models, Journal of the American Statistical Association 108 (503) (2013) 1009-1020.

[29] M. A. Bazaz, M. U. Nabi, S. Janardhanan, A review of parametric model order reduction techniques, in: IEEE International Conference on Signal Processing, Computing and Control (ISPCC), 2012.

[30] J. Lofberg, YALMIP: A toolbox for modeling and optimization in matlab, Online: http : //users.isy.liu.se/johanl/yalmip/ (2014).

[31] IBM, CPLEX Optimizer, Online: http : / / 01.ibm.com/software/commerce/optimization/cplex - optimizer/ (2014).

[32] ETH, Institute for Dynamic Systems and Control, dpm-function, Online: http : //www.idsc.ethz.ch/Downloads/DownloadFiles/dpm (2014). 